\title{
Intervención educativa de enfermería para la rehabilitación de personas con una ostomia
}

\author{
Educative intervention of nursering \\ in the rehabilitation of an ostomized person
}

Lic. Lucía Pat Castillo • Pas. Lic. Enf. Juan Manuel Espinosa Estevez • E.G. María del Pilar Sánchez Bautista • Lic. Otilia Cruz Castañeda • Lic. Marco Antonio Cano Garduño • M.E. Juan Gabriel Rivas •• M.E. Sandra Hernández Corral •.•

\section{Resumen}

Introducción: El incremento del número de personas poseedoras de un estoma, trae consigo la necesidad de contar con personal capacitado para proporcionarle los elementos necesarios para su reintegración a la vida social, familiar y laboral. Objetivo: Analizar el efecto que tiene la intervención educativa de enfermería para la rehabilitación de personas con una ostomia. Metodología: Se trata de un estudio de tipo cuasi-experimental, la muestra se constituyo de 110 personas ostomizadas de cuatro hospitales a las que se les aplicó un instrumento que fue validado por expertos en el área. Para el análisis de datos se utilizó estadística descriptiva e inferencial por medio de la X2. Resultados: En los resultados se observa diferencia estadísticamente significativa entre la intervención educativa y la rehabilitación laboral ( $p=0.000$ ), también se observo diferencia estadísticamente significativa entre la intervención educativa y la rehabilitación social y familiar ( $p=0.000$ ). Discusión: Coincidiendo con Montovani en donde afirma que la falta de información, educación y comunicación son la causa para que el paciente no pueda participar activamente en su autocuidado. De acuerdo con Boccardo se establece que la mayoría de los pacientes ostomizados no retornan totalmente al trabajo pero si parcialmente a sus actividades siendo ésta la parte más difícil de superar. Conclusiones: Se sustenta que la intervención educativa en personas ostomizadas planificada, estandarizada y evaluada, es fundamental para lograr su rehabilitación educativa, laboral, social y familiar. Conclusiones: Se sustenta que la intervención educativa en personas ostomizadas planificada, estandarizada y evaluada, es fundamental para lograr su rehabilitación educativa, laboral, social y familiar.

\footnotetext{
" Hospital Regional "General Ignácio Zaragoza”, Clínica de Ostomias.

"Facultad de estudios Superiores Zaragoza

*** Instituto Nacional de Rehabilitación

Dirección para correspondencia: Lic. Lucia Pat Castillo

Correo electrónico• Lic. Lucía Pat Castillo: luciapatcastillo@hotmail.com
}

RECIBIDO: 18 DE MARZO DE 2010.

ENVIADO A CORRECIONES: 25 DE MARZO DE 2010.

ACEPTADO: 5 DE ABRIL DE 2010.

\section{Palabras \\ Clave: \\ Intervención \\ educativa,}

rehabilitación,

persona ostomizada 


Key
Words:
Intervention
Educative
Rehabilitation
Ostomized person

Introduction: The increase of the number of people, who have a stoma, brings the necessity to have capacitated personal to give them the necessary tools or elements for their reintegration to social and labor life. So that is why it is very important and necessary to analyze the effect that has an educative intervention of nursering in the rehabilitation of people in a kindness context. Method: The method is about a cuasi-experimental and transversal study. The sample was created with the help of one hundred and ten people from four different hospitals. All of these people were given an instrument that was recognized by experts in the area. For the analysis of data, it was used a descriptive statistic of the use of X2. Results: In the results we can observe an important difference between the educative intervention and the labor rehabilitation ( $p=0.000$ ). It was also observed an important statistic difference between the educative intervention and the family and social rehabilitation ( $p .=0.000$ ). Discussion: In agreement with Montovani, the lack of information, education, and communication, prevents the patient from participating actively in their self-care, and according to Boccardo, the majority of ostomized patients, do not fully return to their normal activities. Conclusions: It is supported that the educative participation by plannifying, standarizing and evaluating people is important to obtain their rehabilitation.

\section{INTRODUCCIÓN}

Las unidades médicas de segundo y tercer nivel, actualmente ha aumentado el número de personas a las que se realiza un estoma. ${ }^{1}$

Este aumento trae consigo la necesidad de contar con personal capacitado para proporcionarle los cuidados necesarios y fundamentales para su reintegración a su vida social, familiar y laboral. Montovani et al (1994) sugiere que la falta de información, educación y comunicación son la causa para que el paciente no pueda participar activamente en su cuidado. ${ }^{2}$

La información proporcionada a la persona ostomizada y la familia en el ámbito hospitalario debe fortalecerse a su egreso de acuerdo a la necesidad de cada uno de ellos, por lo cual la educación debe de comenzar desde el momento en que el paciente conoce el tratamiento a seguir de acuerdo a su patología hasta el momento en que pueda ser autosuficiente. ${ }^{3}$

El proceso de educación debe tener en cuenta la edad, la evaluación física, psicológica, los aspectos socioculturales, el grado de temor y el tipo de estoma que se le va a realizar adaptándose a las necesidades de educación de cada persona ostomizada. ${ }^{3,4}$

El manejo temprano del equipo en forma adecuada para cada persona es importante ya que en esta etapa se decide cual es el que utilizará, esto es fundamental para cubrir sus necesidades haciendo su adaptación más fácil a su nuevo estilo de vida, por lo que la selección del equipo y el manejo adecuado del mismo son primordiales para su rehabilitación. ${ }^{5}$

Las complicaciones pueden detener o retardar el proceso de rehabilitación, estas alteraciones deben ser detectadas y manejadas en forma inicial, si la persona poseedora de un estoma recibe la intervención educativa adecuada. ${ }^{5}$

Después de haberse completado la cicatrización y la ostomia funcione normalmente, la mayoría de las personas pueden volver a una dieta normal. Sin embargo después de este tipo de cirugías requieren de información dietética, por lo que las medidas alimentarias son importantes para conseguir un óptimo estado de nutrición $\mathrm{y}$ un correcto funcionamiento del estoma. ${ }^{6,7}$

Todo ser humano va construyendo a lo largo de su vida una imagen única de su cuerpo, el cual se adapta a las costumbres y al medio ambiente en el que vive siempre teniendo en cuenta sus necesidades, por lo que este tipo de cirugía provoca en la persona cambios drásti- 
cos como la pérdida de autonomía, negación al autocuidado, perdida o disminución de la autoestima, miedo, duda, depresión, ansiedad y estrés. Los grupos de autoayuda son importantes para su recuperación ya que la motivación es fundamental para su autocuidado. ${ }^{8,9}$

Hay que recordar que la persona sometida a una ostomia presenta cambios fundamentales y drásticos que le provocan perdida de la autonomía, negación al autocuidado, perdida o disminución de la autoestima, miedo, duda, depresión, ansiedad y estrés. ${ }^{9}$

Por lo que el regreso al trabajo puede ser una etapa difícil ya que la persona ostomizada tiene inseguridad de ir a trabajar con su estoma o la forma en que se puede desenvolver dentro del mismo, como hace referencia Boccardo et al (2002) encontró que la mayoría de los pacientes ostomizados no retornan totalmente al trabajo, apenas se reincorpora parcialmente a las actividades de la vida diaria y las sociales que es lo más difícil, siendo importante la enseñanza impartida por el profesional de enfermería lo cual influye favorablemente en el autocuidado, sin embargo dependerá del grado de instrucción que el paciente reciba para que adopte nuevas conductas durante su cuidado; ya que debe tener conocimientos básicos para comprender sus necesidades y elegir alternativas que ayuden a su recuperación. Un hallazgo importante fue la reincorporación a sus actividades en mayor proporción de los varones (50\%) que de las mujeres (20\%). ${ }^{10}$

En el programa de educación para la salud en pacientes con ostomias urinarias y digestivas, presentado en el V Congreso Nacio-

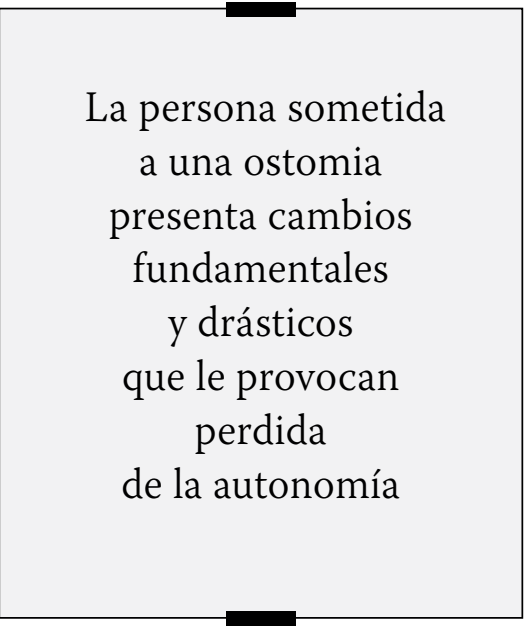

nal de Enfermería en Ostomias, en Pamplona España, mencionan que para conseguir una rápida integración en su entorno social, familiar y laboral la Educación para la Salud (EPS) planificada, estandarizada y evaluada es muy importante. Esta nueva situación precisa adquirir conocimientos y habilidades e implicar al paciente y familia en el autocuidado y propone un enfoque a la educación actuando sobre niveles cognitivos, afectivos y conductuales, proporcionando información y habilidades psicomotoras, para que los pacientes ostomizados puedan modificar su estilo de vida, participando activamente en su autocuidado. ${ }^{11}$

En este mismo contexto Rebagliati et al (2002) menciona que es importante la enseñanza que imparte el profesional de enfermería ya que tiene un efecto favorable en cuanto al autocuidado. ${ }^{12}$

Por lo que la enseñanza que el personal de enfermería provee, es fundamental para estas personas ya que sienta las bases para la rehabilitación y recuperación de su salud; y que a quienes no se les proporciona esta enseñanza difícilmente tendrá la oportunidad de lograr su autocuidado en el área familiar, laboral y social.
Actualmente existen en México diversas clínicas y servicios que proporcionan este cuidado a las personas poseedoras de un estoma, sin embargo, es necesario revisar qué tan efectiva está siendo esta capacitación, por lo que se debe analizar el efecto que tiene la intervención educativa de enfermería en la rehabilitación social, laboral y familiar de la persona ostomizada.

\section{Metodología}

Se trata de un estudio de tipo cuasi experimental que se realizó en el periodo de enero a agosto del 2009.

La muestra se constituyó de 110 personas ostomizadas de las cuales el 50\% el grupo experimental (A) recibió intervención educativa especifica posterior a su cirugía para su rehabilitación en el Hospital Regional General Ignacio Zaragoza del ISSSTE; el otro 50\% pertenece a personas ostomizadas el grupo control (B) de otras unidades médicas de salud del Distrito Federal que no recibieron intervención educativa especifica posterior a la cirugía y que representan el grupo control. La muestra es no probabilística por conveniencia y se determinó por el número de pacientes que cumplió con los criterios de inclusión en ambos grupos; personas con manejo ambulatorio poseedores de una colostomía, ileostomía y/o urostomía después de noventa días de su cirugía, personas que tengan controlada su enfermedad de base y sin secuelas neurológicas o motoras.

La definición conceptual de las variables dentro de este trabajo se estableció de la siguiente manera:

Rehabilitación familiar y social: Se describe cuando la persona ostomizada convive con su familia y 
su núcleo de amigos en los 30 a 60 días posterior a la cirugía, así como el uso de su ropa habitual.

Rehabilitación laboral: Es cuando la persona ostomizada regresa a su trabajo de 30 a 60 días después de la cirugía.

Manejo del equipo: Elección de material de acuerdo a las características que posea el estoma del paciente, así como establecer el procedimiento de colocación, cuidado y retiro del mismo.

Y la operacionalización de estas variables se estableció de la siguiente forma:

1. Definitivamente si

2. Algunas veces

3. Rara vez

4. Definitivamente no
Se estructuró un instrumento con 13 ítems con un apartado de respuestas dicotómicas y otro con escala tipo liker, validado por 11 enfermeras enterostomales de diferentes unidades de salud, aplicado a las personas ostomizadas el día programado para su dotación de material en cada unidad de salud participante.

El análisis de los resultados se elaboró con una base de datos en el programa SPSS V15, utilizando como unidad de análisis estadístico descriptivo frecuencias y porcentajes, para el análisis inferencial la X2 presentando los resultados en cuadros.

\section{Resultados}

Como se puede observar (cuadro1) en los dos grupos estudiados no existe diferencia significativa en cuanto al sexo, grupo de edades, tipo de estoma y actividad laboral, por lo tanto es una muestra homogénea compartiendo las mismas características para los datos demográficos.

De los pacientes que recibieron intervención educativa se rehabilitaron el $89 \%$ en el ámbito social y familiar, $69 \%$ se rehabilito laboralmente y el $85 \%$ maneja su equipo, mientras que el grupo que no recibió intervención educativa el $80 \%$ no se rehabilito en lo social y familiar, el 75\% no se rehabilito laboralmente y el $53 \%$ no maneja su equipo (cuadro 2).

En la relación propuesta de la intervención educativa y la rehabilitación social, familiar y laboral la diferencia fue estadísticamente significativa con un valor de $\mathrm{p}=0.000$.

\section{Cuadro 1. Variables demográficas}

\begin{tabular}{llll}
\hline & Grupo experimental & & General \\
& $\mathbf{N}=\mathbf{( 5 5 )}$ & Grupo N=(55) & $\mathbf{N}=(\mathbf{1 1 0})$ \\
\hline Femenino & $32(58 \%)$ & $33(60 \%)$ & $65(59 \%)$ \\
Masculino & $23(42 \%)$ & $22(40 \%)$ & $45(41 \%)$ \\
Edad 7 a 12 años & $1(2 \%)$ & 0 & $1(1 \%)$ \\
De 13 a 18 años & $1(2 \%)$ & $1(2 \%)$ & $2(2 \%)$ \\
De 19 a 50 años & $16(29 \%)$ & $17(31 \%)$ & $33(30 \%)$ \\
De 51 a 80 años & $35(64 \%)$ & $35(64 \%)$ & $70(63 \%)$ \\
Más de 81 años & $2(4 \%)$ & $2(4 \%)$ & $4(4 \%)$ \\
Colostomia & $40(73 \%)$ & $37(67 \%)$ & $77(70 \%)$ \\
Ileostomía & $10(18 \%)$ & $11(20 \%)$ & $21(19 \%)$ \\
Urostomía & $5(9 \%)$ & $7(13 \%)$ & $12(11 \%)$ \\
Trabaja & $24(44 \%)$ & $21(38 \%)$ & $45(41 \%)$ \\
No trabaja & $8(16 \%)$ & $13(24 \%)$ & $21(19 \%)$ \\
Actividades del hogar & $21(38 \%)$ & $21(38 \%)$ & $42(38 \%)$ \\
Estudiante & $2(4 \%)$ & 0 & $2(2 \%)$ \\
\hline Euente: instrumento de intervención educativa de enfermería para el paciente ostomizado, 2009. & \\
\hline
\end{tabular}




\section{Cuadro 2. Intervención educativa y rehabilitación social, familiar, laboral y manejo del equipo.}

\begin{tabular}{|c|c|c|c|c|c|c|}
\hline \multirow{2}{*}{$\begin{array}{l}\text { Intervención } \\
\text { educativa }\end{array}$} & \multicolumn{6}{|c|}{ Rehabilitación } \\
\hline & \multicolumn{2}{|c|}{$\begin{array}{l}\text { Social y familiar } \\
\text { Si No }\end{array}$} & \multicolumn{2}{|c|}{ Laboral } & \multicolumn{2}{|c|}{ Manejo del equipo } \\
\hline $\mathrm{Si}$ & $49(89 \%)$ & $6(11 \%)$ & $38(69 \%)$ & 17 (31\%) & $47(85 \%)$ & $8(15 \%)$ \\
\hline No & $11(20 \%)$ & $44(80 \%)$ & $14(25 \%)$ & $41(75 \%)$ & $26(47 \%)$ & $29(53 \%)$ \\
\hline
\end{tabular}

\section{Discusión}

De acuerdo a los resultados obtenidos en la rehabilitación social y familiar se encontró que las personas que recibieron intervención educativa se rehabilitaron en un alto porcentaje, a diferencia del grupo que no la recibió, dato importante ya que la reintegración de estas personas a su núcleo social y familiar es fundamental para que ellos continúen con su vida en forma normal y no se sientan excluidos, tal como se estableció en las investigaciones presentadas en el V Congreso Nacional de Enfermería en Ostomias en Pamplona España, donde se puntualizó que la educación planificada, estandarizada y evaluada es importante para la rápida integración de la persona ostomizada a su entorno social familiar y laboral.

Con respecto a la rehabilitación laboral, encontramos que el grupo experimental se reintegró en un alto porcentaje a su trabajo y dentro del grupo control las personas que se reintegraron fue por la necesidad económica a pesar de ameritar incapacidades médicas, situación que se manejo en el estudio realizado en noviembre del 2002 en
Lima Perú por Boccardo en donde establece que la mayoría de los pacientes ostomizados no retornan totalmente al trabajo pero si parcialmente a sus actividades siendo ésta la parte más difícil de superar.

En cuanto al equipo se encontró que las personas que recibieron intervención educativa lo manejan adecuadamente, en contraste con el que no la recibió; cabe mencionar que este último grupo presento una serie de complicaciones en la piel periostomal, dato muy importante, ya que el equipo es una de las herramientas fundamentales para que la persona ostomizada se rehabilite, como se menciona en la investigación realizada en el año de 1994 por Montovani titulada "El proceso interactivo de vivir con el estoma propiciando la enseñanza y autoaprendizaje", donde sugiere que la falta de información, educación y comunicación son la causa para que el paciente no pueda participar activamente en su autocuidado.

\section{Conclusiones}

De acuerdo a los datos obtenidos en esta investigación se infiere que la función específica de la enfermera terapista enterostomal en cuanto a la intervención educativa para la rehabilitación de la persona poseedora de un estoma de eliminación cobra mayor importancia, así como el contar con un manual especifico de capacitación para la persona ostomizada.

Por otro lado el contar con una clínica de ostomias redundara no solo en la atención que se le proporciona a la persona ostomizada si no que también se traduce en la disminución de costos para las diferentes instituciones y el paciente.

\section{REFERENCIAS BIBLIOGRÁFICAS}

1. Ortiz H, Marti RJ, Foulkes B. Indicaciones y cuidados de los estomas: Técnicas quirúrgicas. $1^{\circ}$ ed. Barcelona, España; 1994. p. 107-30.

2. Espinoza HM. Conocimientos y prácticas sobre el autocuidado que tienen los pacientes colostomizados que asisten a la consulta de enfermería del Hospital Nacional Edgardo Rebagliati Martins. Disponible en URL http://www. minsa.gob.ni/enfermeria/doc_ inter/T_completo02.pdf. 2002. p. $47-62$ 
3. Molina GAM, Guisado CI, Valenciano MA. Atención integral al paciente ostomizado: Elección de procedimientos terapéuticos. $1^{\circ}$ ed. España; 1992. p. 107-33.

4. Cruz CO, Pat CL, González RMC, Elizondo VJF. Manual de procedimientos técnicos de la clínica de ostomias y heridas: Técnicas de atención al paciente con estomas intestinales. México; 2004. p. 3- 10.

5. Canaval GE, Londoño ME, Milena HA. Guía de enfermería para el cuidado de la persona adulta con estoma. Guías ACOFAEN. Biblioteca Las casas, 2005; 1. Disponible en URL: http://www.indexf.com/ lascasas/documentos/lc0026.php

6. Cuidados del paciente ostomizado. Zumarraga. Hospital de Zumarraga. Disponible en URL http:// www.kideitu.euskadi.net/contenidos/informacion/hzum_paciente_ ostomizado/eu_hzum/adjuntos/ ostomizatu_paziente_zainketa. pdf. p. 3-4
7. Oliva AC, Mederos CO, Barrera OJ, Da CJ. Recomendaciones nutricionales a apacientes ostomizados. Disponible en URL: http://www.sld.cu/galerias/pdf/ uvs/cirured/recomendaciones_ nutricionales_a_pacientes_ostomizados.pdf. p. 5-7

8. Claudio G, Patkan M, Torres V, Merino C, Pascal S, Moriggia I, et al. El paciente ostomizado en el entorno de una asociación. Disponible en URL: http://www.acosur.org.ar/ Revista/archivos/v2n4/El\%20ostomizado\%20controlado.pdf. Bilbao; Dic. 2005.2 (4). p. 201-11.

9. Romaguera A. R. El paciente socio sanitario ostomizado. Disponible en URL:http://www.enfervalencia. org/ei/79/articulos-cientificos/5. pdf Enfermería Integral Septiembre 07. p. 22.

10. Zambrano GJ y Pajuelo VR. Beneficios de la técnica de irrigación de colostomía de los pacientes colostomizados en el servicio de cirugía del Hospital Víctor Ramos Guar- dia Huaraz. Revista Científica De Enfermería - Recién. pp. 62-71.

11. Arrontes CG, Frutos MR, González RV, Ramírez BM, Zabala LI, Hernando LA, et al. Necesidades de cuidados y calidad de vida de los pacientes ostomizados. Educare21 2008; 49. URL disponible en: http://preview.enfermeria21.com/ educare/secciones/casosclinicos/

12. Guindano GL; Soteras RM; Beortegui UE; Canga AN y Fuertes RC. Programa de educación para la salud en pacientes con ostomias urinarias y digestivas. V Congreso $\mathrm{Na}$ cional de Enfermería en Ostomias. Disponible en URL http://www. docstoc.com/docs/22739983/ PROGRAMA-DE-EDUCACI?NPARA-LA-SALUD-EN-PACIENTES-CON/. Pamplona, España. p. 104-110. 